\title{
Emergence of a dark force in corpuscular gravity
}

\author{
M. Cadoni, ${ }^{1,2, *}$ R. Casadio, ${ }^{3,4, \dagger}$ A. Giusti, ${ }^{3,4,5, \$}$ and M. Tuveri ${ }^{1,2, \S}$ \\ ${ }^{1}$ Dipartimento di Fisica, Università di Cagliari, Cittadella Universitaria, 09042 Monserrato, Italy \\ ${ }^{2}$ I.N.F.N., Sezione di Cagliari, Cittadella Universitaria, 09042 Monserrato, Italy \\ ${ }^{3}$ Dipartimento di Fisica e Astronomia, Università di Bologna, Via Irnerio 46, 40126 Bologna, Italy \\ ${ }^{4}$ I.N.F.N., Sezione di Bologna, IS_FLAG, Via Berti Pichat 6/2, 40127 Bologna, Italy \\ ${ }^{5}$ Arnold Sommerfeld Center, Ludwig-Maximilians-Universität, \\ Theresienstraße 37, 80333 München, Germany
}

(Received 6 February 2018; published 28 February 2018)

\begin{abstract}
We investigate the emergent laws of gravity when dark energy and the de Sitter space-time are modeled as a critical Bose-Einstein condensate of a large number of soft gravitons $N_{\mathrm{G}}$. We argue that this scenario requires the presence of various regimes of gravity in which $N_{\mathrm{G}}$ scales in different ways. Moreover, the local gravitational interaction affecting baryonic matter can be naturally described in terms of gravitons pulled out from this dark energy condensate (DEC). We then explain the additional component of the acceleration at galactic scales, commonly attributed to dark matter, as the reaction of the DEC to the presence of baryonic matter. This additional dark force is also associated to gravitons pulled out from the DEC and correctly reproduces the modified Newtonian dynamics (MOND) acceleration. It also allows for an effective description in terms of general relativity sourced by an anisotropic fluid. We finally calculate the mass ratio between the contribution of the apparent dark matter and the baryonic matter in a region of size $r$ at galactic scales and show that it is consistent with the $\Lambda \mathrm{CDM}$ predictions.
\end{abstract}

DOI: 10.1103/PhysRevD.97.044047

\section{INTRODUCTION}

One of the major ideas triggering recent theoretical progress about the gravitational interaction is that of emergent gravity: the classical space-time structure and gravity emerge together from an underlying microscopic quantum theory [1-3]. The power of this emergent paradigm is that it must depend loosely on the details of the underlying microscopic theory and it is essentially determined by its fundamental quantum nature.

The notion of emergent gravity is quite general and it has been used in several different contexts $[1,2,4-10]$. In this paper we want to focus on two main realizations of this idea, which have recently attracted a lot of attention. The first one uses the entanglement of microscopic quantum states as the origin of space-time geometry. This route historically starts from the discovery of the Bekenstein-Hawking $(\mathrm{BH})$ entropy area law [11] and goes through the development of the AdS/CFT correspondence [12] and the RyuTakayanagi formula, where it clearly appears that the $\mathrm{BH}$ formula is related to the quantum entanglement of the vacuum [13]. Subsequently, it was also realized that quantum entanglement could explain the connectivity of classical

\footnotetext{
*mariano.cadoni@ca.infn.it

†casadio@bo.infn.it

agiusti@bo.infn.it

\$matteo.tuveri@ca.infn.it
}

space-time [14], and that the linearized Einstein equations can be derived from quantum information principles [15]. The second main realization of the idea that gravity is emergent uses the notions of quantum compositeness and classicalization [6,7]. Gravitational systems, such as black holes and cosmological spaces, can be described as a composite quantum system of a large number $N_{\mathrm{G}}$ of soft gravitons. It has been shown that these gravitational systems exhibit properties of a Bose-Einstein condensate (BEC) at the quantum critical point. Moreover, the usual classical space-time structure emerges in the limit $N_{\mathrm{G}} \rightarrow \infty$ of this picture [6,7]. This corpuscular realization of the paradigm of emergent gravity has been also successfully used to describe Hawking radiation $[16,17]$ and inflation $[7,18,19]$.

One common intrinsic feature of both the above realizations of emergent gravity is that of holography. The intrinsic holographic nature of the quantum entanglement approach is evident in the Ryu-Takayanagi derivation of the entanglement entropy and, more in general, in the quantum information picture of black holes and cosmological horizons [3,13]. The same holographic nature is at the heart of the corpuscular approach, which is based on the fact that the number $N_{\mathrm{G}}$ of soft gravitons in a BEC at the critical point scales, in terms of the size $r$ of the system, as ${ }^{1}$

\footnotetext{
${ }^{1}$ We shall use units with $c=1$ but display explicitly the Planck constant $\hbar=\ell_{\mathrm{p}} m_{\mathrm{p}}$ and Newton constant $G_{\mathrm{N}}=\ell_{\mathrm{p}} / m_{\mathrm{p}}$.
} 


$$
N_{\mathrm{G}} \sim \frac{r^{2}}{\ell_{\mathrm{p}}^{2}}
$$

where $\ell_{\mathrm{p}}$ is the Planck length. The holographic character of this description is also very important for understanding the quantum information counted by the $\mathrm{BH}$ entropy (see the complementary, firewall and $\mathrm{ER}=\mathrm{EPR}$ discussion $[20,21]$ ) and for the description of black holes as the BEC of gravitons at the critical point $[6,7]$.

The emergent gravity scenario also provides a connection between the microscopic ultraviolet (UV) scale $\ell_{\mathrm{p}}$ and the infrared (IR) cosmological scale $L=H^{-1}$ of gravity (here $H$ is the Hubble parameter and $L$ the Hubble scale). In the quantum entanglement setup, the entropy associated to the de Sitter (dS) space-time can be explained, similarly to the $\mathrm{BH}$ entropy, as a long range entanglement connecting bulk excitations with the dS horizon [3]. In the corpuscular setup, both black holes and our observable Universe are "maximally classical" systems, i.e. BEC at the critical point satisfying the relation (1.1) with $r=R_{\mathrm{H}}$ (the Schwarzschild radius) and $r=L$, respectively.

The success of emergent gravity in describing the holographic regimes of gravity shown in Eq. (1.1), i.e. black holes and the $\mathrm{dS}$ universe, gives a strong motivation to use it also at intermediate scales, i.e. at galactic scales. It is quite evident that the behavior of the gravitational interaction at these scales cannot be simply described by the maximally packing condition (1.1). On the other hand, explaining the phenomenology of gravity at galactic scales has been one of the main motivations for introducing dark matter [22-24] and the $\Lambda C D M$ model [25-28]. One is therefore led to expect that the application of the emergent gravity scenario at galactic scales may hold the key to understanding the dark matter mystery.

In a fully emergent gravity scenario, such as the one we consider in this work, in which matter and space-time are intimately related, the existence of a form of matter different from the baryonic one is conceptually weird. Moreover, we recall that the $\Lambda \mathrm{CDM}$ model is not completely satisfactory also from the observational point of view, both at the level of galaxies and galaxy clusters [2932]. If one does not assume the existence of dark matter, a crucial challenge for every model of emergent gravity is the explanation of galaxy rotation curves $[33,34]$ and the TullyFisher relation [35] between the velocity of stars far away from the galactic center and the total baryonic mass $m_{\mathrm{B}}$ contained in the galaxy.

In the framework of modified Newtonian dynamics (MOND) [36,37], the Tully-Fisher relation is explained assuming that, at distances outside the galaxy's inner core, the gravitational acceleration experienced by a test particle is given by

$$
a_{\mathrm{MOND}}(r)=\sqrt{\frac{a_{\mathrm{B}}(r)}{6 L}}
$$

where

$$
a_{\mathrm{B}}(r)=\frac{G_{\mathrm{N}} m_{\mathrm{B}}(r)}{r^{2}}
$$

is (minus) the Newtonian radial acceleration that would be caused by the baryonic mass $m_{\mathrm{B}}=m_{\mathrm{B}}(r)$ inside the radius $r$.

A first step to explain Eq. (1.2) in the framework of gravity emerging from quantum entanglement has been undertaken in Ref. [3]. In that work, it was shown that, when applied at galactic scales, the laws of emergent gravity contain an additional dark gravitational force, which may explain the phenomenology commonly attributed to dark matter and reproduce the MOND acceleration (1.2). Following Verlinde [3], the long range entanglement connecting bulk excitations with the dS horizon (i.e. the positive dark energy) generates a (thermal) volume contribution to the entanglement entropy and a subsequent competition between area and volume laws. This can be seen as an elastic response of the dark energy medium to the presence of baryonic matter which, in turn, implies an additional dark gravitational force correctly reproducing the MOND acceleration [3].

The purpose of this paper is to explain the generation of an additional dark gravitational force at galactic scales and derive the MOND acceleration (1.2) using the corpuscular approach to emergent gravity. This will be done by developing further and generalizing some ideas presented in Ref. [38], where an effective fluid approach for the dark energy condensate (DEC) of soft gravitons permeating the universe has been used.

We shall begin with a critical discussion of various regimes of gravity in the corpuscular scenario. In Sec. II, we will start by arguing that describing dark energy as a critical BEC of soft gravitons (the DEC) implies not only the presence of a nonextensive regime of gravity satisfying Eq. (1.1), but also of an extensive regime in which $N_{\mathrm{G}} \sim r^{3} /\left(L \ell_{\mathrm{p}}^{2}\right)$. The local gravitational interaction with baryonic matter can then be naturally described in terms of gravitons pulled out from the DEC. We will first consider, in Sec. III, baryonic matter in the diluted approximation, when the local reaction of the condensate to the presence of baryonic matter can be (ideally) neglected. We will then proceed by describing what happens when we go beyond the diluted approximation and baryonic matter begins to clump. We will show that, in this regime, the reaction of the DEC to the presence of baryonic matter is also associated to gravitons pulled out from the DEC. They generate an additional gravitational dark force on baryonic probe sources, which correctly reproduces the MOND acceleration and allows for an 
effective description in terms of general relativity (GR) sourced by an anisotropic fluid. In Sec. IV, we will also compute the ratio between the apparent dark matter and the baryonic component to the mass density and show that it is consistent with the $\Lambda \mathrm{CDM}$ result for the present abundance of different kinds of matter. We finally conclude with some considerations about future developments in Sec. V.

\section{QUANTUM COMPOSITENESS AND THE SCALING OF GRAVITON NUMBER}

Our starting point is that the true quantum nature of gravity cannot be fully neglected in our present universe, even at astrophysical and cosmological scales, and the geometric description given by Einstein gravity (or modifications thereof) should only emerge in suitable regimes and for specific observables. In particular, it has been conjectured that the quantum state of our Universe could be thought of as a BEC [7] containing a certain number $N_{\mathrm{G}}$ of (very soft and virtual) gravitons with typical energy $\varepsilon_{\mathrm{G}}$, very much like the gravitational field of a black hole [6]. The presence of baryonic matter must affect the quantum state of this BEC of gravitons and, at least in some crude approximation, one can then expect that an energy balance, akin to the Hamiltonian constraint of GR, holds in the form

$$
H_{\mathrm{B}}+H_{\mathrm{G}}=0,
$$

where $H_{\mathrm{B}}$ is the matter energy and $H_{\mathrm{G}}$ the analogue quantity for the graviton state.

It is now crucial that our present Universe appears to be mostly driven by dark energy, and as such it is characterized by the Hubble radius,

$$
L=H^{-1},
$$

of the visible portion. Furthermore, the presence of baryonic matter (stars and planets) defines a typical size $R_{\mathrm{B}}$, around which gravity is well approximated by Newtonian physics. These two length scales satisfy the hierarchy

$$
R_{\mathrm{H}} \ll R_{\mathrm{B}} \ll L,
$$

where $R_{\mathrm{H}}=2 G_{\mathrm{N}} m_{\mathrm{B}}$ is the Schwarzschild radius of a source of baryonic mass $m_{\mathrm{B}}$. The quantum state of gravity should entail such scales. In particular, we expect to identify different regimes of gravity for each scale from the way both the number of gravitons $N_{\mathrm{G}}$ and their typical energy $\varepsilon_{\mathrm{G}}$ scale with the mass $m=m(r)$ and the size $r$ of the region we are considering.

In the corpuscular description, one is mainly concerned with self-gravitating systems, i.e. compact sources of typical size $R_{\mathrm{B}}$. To this class belong both marginally bound systems, which are described by BEC at the critical point (black holes) and nonmarginally bound systems (compact stars, horizonless objects) which are described by BEC away from the critical point. In terms of the graviton coupling $\alpha \simeq \ell_{\mathrm{p}}^{2} / r^{2}$ the two regimes respectively correspond to $\alpha=1 / N_{\mathrm{G}}$ and $\alpha<1 / N_{\mathrm{G}}$ [6].

In terms of the Hamiltonian constraint (2.1) the marginally bound condition corresponds to systems for which the mass is equal to the graviton interaction energy [39]. At small scales, i.e. for $r$ of the order of the size of compact sources, the number of gravitons $N_{\mathrm{G}}$ affected by the presence of matter sources can be obtained by describing the Newtonian (and first post-Newtonian $[39,40]$ ) potential by means of a quantum coherent state, for which one generically finds a quadratic scaling of $N_{\mathrm{G}}$ with the mass [6],

$$
N_{\mathrm{G}} \sim \frac{m_{\mathrm{B}}^{2}}{m_{\mathrm{p}}^{2}},
$$

where $m_{\mathrm{B}}$ is the mass of the localized baryonic source. Since the (negative) Newtonian energy is given by

$$
U_{\mathrm{N}} \simeq N_{\mathrm{G}} \varepsilon_{\mathrm{G}} \simeq-\frac{G_{\mathrm{N}} m_{\mathrm{B}}^{2}}{r},
$$

the typical energy of the individual (virtual) quanta is again given by the Compton relation

$$
\varepsilon_{\mathrm{G}} \simeq-\frac{\ell_{\mathrm{p}} m_{\mathrm{p}}}{r}
$$

and, using the mass/radius relation for black holes, $m_{\mathrm{B}} \simeq r=R_{\mathrm{H}}$, Eq. (2.5) implies a holographic scaling with $r$, namely

$$
N_{\mathrm{G}} \sim \frac{r^{2}}{\ell_{\mathrm{p}}^{2}} \sim-\frac{1}{\rho_{\mathrm{H}}},
$$

where $\rho_{\mathrm{H}}$ is the (negative) graviton energy density around a black hole.

Notice that, for nonmarginally bound gravitational systems, the scaling relation (2.4) still holds [39,40], but the holographic (2.7) does in general not. The corpuscular description can be generalized to cosmological space-times [7] in the absence of baryonic matter. In this framework, the dS universe of size $L$, sourced by a constant dark energy density $\rho_{\Lambda}$, can be described, similarly to a black hole, as a critical BEC [41]. In fact, the main feature of the dark energy sourcing the dS space-time, namely that it satisfies the vacuum equation of state $p=-\rho_{\Lambda}$, is naturally realized in a BEC, as was shown in Refs. [42-45].

An ideal universe of size $L$ solely containing selfcoupled gravitons as a description of vacuum (dark) energy should behave like the de Sitter space-time. In GR, one then needs a cosmological constant term, or constant vacuum energy density $\rho_{\Lambda}$, so that the Friedman equation reads 


$$
H^{2} \equiv\left(\frac{\dot{a}}{a}\right)^{2} \simeq G_{\mathrm{N}} \rho_{\Lambda}
$$

Upon integrating on the volume inside the Hubble radius (2.2), we obtain

$$
L \simeq G_{\mathrm{N}} L^{3} \rho_{\Lambda} \simeq G_{\mathrm{N}} m_{\Lambda}
$$

This relation looks like the expression of the horizon radius for a black hole of ADM mass $m_{\Lambda}$, which has led to the conjecture that the dS space-time could likewise be described as a condensate of gravitons [7,41]. One can in fact introduce a corpuscular description on assuming that the (soft virtual) graviton self-interaction gives rise to a condensate of $N_{\Lambda}$ gravitons of typical Compton length equal to $L$ [7], so that the total (positive dark) energy

$$
m_{\Lambda} \simeq N_{\Lambda} \varepsilon_{\Lambda} \simeq N_{\Lambda} \frac{\ell_{\mathrm{p}} m_{\mathrm{p}}}{L}
$$

and, from Eq. (2.9), it follows immediately that

$$
N_{\Lambda} \sim \frac{m_{\Lambda}^{2}}{m_{\mathrm{p}}^{2}}=\frac{L^{2}}{\ell_{\mathrm{p}}^{2}},
$$

which shows that one needs a huge $N_{\Lambda} \gg 1$ for a macroscopic universe. Note also that we have

$$
\rho_{\Lambda} \sim \frac{m_{\Lambda}}{L^{3}} \sim \frac{1}{N_{\Lambda}}
$$

so that the number of gravitons in the vacuum increases for smaller vacuum energy, and

$$
L \sim m_{\Lambda} \sim \frac{1}{\sqrt{\rho_{\Lambda}}} .
$$

\section{A. Holographic regimes of gravity}

We have shown that black holes and the dS universe can be described by a critical BEC of gravitons that we dubbed DEC. We have also seen that criticality for the BEC implies the holographic scalings (2.7) and (2.11) for $N_{\mathrm{G}}$. Given that all the gravitons are packed in the ground state, the entropy of the DEC is given by $N_{\mathrm{G}}$, implying that Eqs. (2.7) and (2.11) are equivalent to the $\mathrm{BH}$ area law. ${ }^{2}$

Equations (2.4) and (2.11) define the holographic regimes of gravity: for volumes of both cosmological (in absence of baryonic matter) and Newtonian size, one can argue that the relevant number of gravitons scales holographically, that is

\footnotetext{
${ }^{2}$ Factors of order 1 will be usually neglected unless necessary.
}

$$
N_{\mathrm{G}}^{A}(r) \sim \frac{m^{2}(r)}{m_{\mathrm{p}}^{2}} \sim \frac{r^{2}}{\ell_{\mathrm{p}}^{2}}, \text { for } r \simeq L \text { and } r \simeq R_{B},
$$

where $m=m(r)$ is an appropriate mass function inside the volume. More precisely, $N_{\mathrm{G}}(L)$ can be viewed as the total number of gravitons inside the visible universe, whereas $N_{\mathrm{G}}\left(R_{\mathrm{B}}\right)$ is the number of gravitons that respond locally to the presence of the baryonic sources of mass $m_{\mathrm{B}}$, by changing their energy from $\varepsilon_{\mathrm{G}}(L)$ to some $\varepsilon_{\mathrm{G}}\left(R_{\mathrm{B}}\right)<\varepsilon_{\mathrm{G}}(L)$ in order to enforce the Newtonian dynamics. The holographic scaling (2.14) therefore applies to two very different, albeit equally nonextensive, regimes of gravity.

The holographic scaling relations (2.7) and (2.4) were first found for black holes [7], and only Eq. (2.4) was then shown to hold for general compact sources in Refs. [39,40]. From Eq. (2.4), it follows that we get the $\mathrm{BH}$ area law (2.14) in the regime where the relevant mass $m=m(r)$ of the condensate scales linearly with the size $r$ of the source.

The holographic regime of gravity holds for sure in the case of black holes and the de Sitter space, and we assume that Eq. (2.14) also remains a very good approximation at all typical scales $r$ for which gravity is well described by GR. This assumption is based on the fact that the holographic nature of gravity is a generic consequence of the Einstein-Hilbert action. Note, however, the change in sign of the graviton energy from the positive cosmological mass (2.10) to the negative Newtonian energy (2.5): this is a clear signal that the two holographic regimes, at small and very large scales, respectively, are indeed different, which suggests that at intermediate scales the behavior of gravity deviates from the holographic description, as we will see in the next section.

Before we proceed, a word of caution is in order: since the gravitons in the condensate are considered as virtual (nonpropagating) modes, their number $N_{\mathrm{G}}$ is not directly observable, nor is their individual energy $\varepsilon_{\mathrm{G}}$. In fact, one can think of these quantities as convenient intermediate variables which will not appear in our final expression for the matter dynamics. These gravitons could however become observable if they are scattered off the coherent state, for instance by their self-interaction, which leads to the depletion of the DEC. This effect produces the Hawking radiation around black holes [6] and primordial perturbations during inflation $[7,46]$, but will be totally neglected in this work.

\section{B. Extensive regime of gravity}

There are several reasons, coming both from the microscopic and from the emergent space-time description, for arguing that the holographic regime (2.14) of gravity cannot hold throughout the whole range of scales (2.3). In particular, this implies the existence of a new infrared scale $R_{\mathrm{H}}<r_{0}<L$, where the behavior of gravity deviates from the holographic description. 
The first indication comes from the fact that the two holographic regimes at small and very large scales, although satisfying the same scaling relation (2.14), are indeed different. We recalled above that the graviton energy changes in sign going from the positive cosmological mass (2.10) to the negative Newtonian energy (2.5). This implies that the two holographic regimes must be connected by a mesoscopic phase, in which gravity may deviate from the holographic behavior (2.14).

The second indication comes from Verlinde's argument about the pattern of entanglement entropy in dS space [3]. Unlike black holes, the dS space-time must contain a thermal volume contribution to the entanglement entropy, coming from very low energy modes. In our description of the dS space-time, this implies an extensive term for the graviton number associated with the DEC.

The third and strongest indication comes from the fact that, locally, without baryonic matter, the DEC of the dS space-time has a constant energy density characterized by an extensive behavior. In fact, at galactic scales, we cannot consider the cosmic condensate as a whole, but just as a medium with (positive) constant energy density $\rho_{\mathrm{G}}$ equal to the cosmological value (2.12), that is

$$
\rho_{\mathrm{G}} \simeq \rho_{\Lambda} \sim \frac{m_{\mathrm{p}}}{L^{2} \ell_{\mathrm{p}}}
$$

The total graviton energy inside a region of size $r$ is therefore given by

$$
m_{\mathrm{G}}(r) \simeq \frac{4 \pi}{3} \rho_{\Lambda} r^{3} \sim \frac{m_{\mathrm{p}} r^{3}}{L^{2} \ell_{\mathrm{p}}}=N_{\mathrm{G}}(r) \varepsilon_{\Lambda} .
$$

The number of gravitons contained in this spherical region is therefore an extensive quantity, scaling as the volume,

$$
N_{\mathrm{G}} \simeq \frac{r^{3}}{L \ell_{\mathrm{p}}^{2}} \sim \frac{m_{\mathrm{G}}(r)}{m_{\mathrm{p}}}
$$

where we again assumed the Compton relation $\varepsilon_{\Lambda} \sim$ $\ell_{\mathrm{p}} m_{\mathrm{p}} / L$ from Eq. (2.10). A crucial check for the validity of the scaling relation (2.17) is that it correctly reproduces the cosmological relation (2.11) precisely for $r=L$.

We are therefore led to assume that, if baryonic matter is totally neglected, at the intermediate scales $R_{\mathrm{B}} \ll r \ll L$, the graviton state is approximately described by the extensive regime (2.17); i.e. it is ruled by the extensive regime of gravity:

$$
N_{\mathrm{G}}^{V}(r) \sim \frac{m}{m_{\mathrm{p}}} \sim \frac{r^{3}}{L \ell_{\mathrm{p}}^{2}}, \quad \text { for } R_{\mathrm{B}} \ll r \ll L .
$$

This behavior will be argued to interpolate somehow between the two (different) holographic regimes (2.14) at $r \simeq L$ and $r \simeq R_{\mathrm{B}}$. One of the main results we will present here is that it is the tension between the two scalings (2.14) and (2.18) that leads to deviations from the local Newtonian dynamics [3]: the response of the graviton condensate to the presence of baryonic matter makes both the holographic and the extensive regimes important at galactic scales.

The physical picture behind this corpuscular description is again similar to Verlinde's [3]. For compact sources of size $R_{\mathrm{B}} \simeq R_{\mathrm{H}}$ and at cosmological scales $L$, gravity allows for a corpuscular description in which it is described by a critical BEC of gravitons. The effective theory in these two regimes is $\mathrm{GR},{ }^{3}$ whose peculiar nonextensive, holographic character is encoded by the relations (2.7) and (2.11). Notice that these two regimes correspond to length scales differing by several orders of magnitude (about 60 if we take $r=\ell_{\mathrm{p}}$ and $r=L$ ), and the same holds for the graviton wavelengths in the two regimes.

A specific merit of the corpuscular picture that we started to build is however that these two holographic regimes are truly different, as the relation (2.14) refers to the total number of gravitons in the cosmological condensate for $r \simeq L$, whereas it only counts the number of gravitons affected by the local matter sources for $r \simeq R_{\mathrm{B}} \gtrsim R_{\mathrm{H}}$. We recall once more that the difference is clearly signaled by the opposite signs of $\varepsilon_{\Lambda}>0$ and $\varepsilon_{\mathrm{B}}<0$.

At intermediate scales the condensate has the intrinsic extensive behavior (2.17), which is a peculiar feature of thermalization processes (corresponding to the slow dynamics of glassy systems in Verlinde's description). Strictly speaking the graviton number $N_{\mathrm{G}}^{V}$ inside a spherical region is not physically measurable. In fact $N_{\mathrm{G}}^{V}$ is not conserved and, for a small region, it is expected to have large relative fluctuations. For regions of galactic or cosmological size, the relative fluctuations are small but we can hardly conceive of a physical process apt to measure $N_{\mathrm{G}}^{V}$. On the other hand, our final results are independent from $N_{\mathrm{G}}^{V}$ and we do not need to be concerned about its measurability.

In principle, there could be concerns about the impact that an extensive, volume-scaling term for $N_{\mathrm{G}}$ can have on the cosmological evolution, in particular for late-time cosmology. At late times, the cosmological dynamics is described by the holographic regime characteristic of the dS space-time as discussed above. Actually, it has been recently shown by Carroll et al. [47] that this is a quite general result. Assuming the validity of a generalized second law of thermodynamics and that the entropy increases up to a finite maximum value, any RobertsonWalker space-time must approach a dS space-time in the future, independently of the gravitational dynamics and matter content of the universe. In their argument, Carroll et al. assume the presence of a constant density term in the

\footnotetext{
${ }^{3}$ Since the universe is expanding, one might argue that the cosmological description is in fact closer to a modified $f(R) \simeq R^{2}$ theory of gravity [40].
} 
generalized entropy, which has the same form of our extensive term (2.17). However, they show that at late times, i.e. for large values of the scale factor, this term is subleading with respect to the holographic one, the latter approaching a constant value and scaling like the area of the dS horizon. Translated in our corpuscular description, this means that our extensive term (2.17) plays a role at intermediate galactic scales, but becomes completely irrelevant for the late-time cosmological evolution.

\section{Baryonic matter and the emergence of a dark force}

So far we have considered the cosmological condensate without baryonic matter. One could just consider baryonic matter always existed inside the DEC, initially in a very diluted form, so that its effect on the gravitons of the cosmological BEC was initially negligible. In time, the baryonic matter clumped and started affecting the DEC locally, which is the situation we find in the universe today. In particular, the presence of local baryonic sources pulls out gravitons from the DEC, which give rise to the local gravitational forces. Alternatively, the simplest way to introduce baryonic matter in our scenario is to assume that it arises as bound states in the DEC, i.e. to consider it as produced by gravitons pulled out from the cosmological condensate at the typical matter scales $R_{\mu}$, where $\mu$ denotes the mass of single pointlike matter sources. This may occur owing to density perturbations in the BEC. A uniform, spherically symmetric overdensity region of the BEC is isotropically compressed, because pressure gradients act only on the surface of the sphere, generating a compact source of baryonic matter, which can itself be described by a noncritical BEC or by critical BEC if the critical density is reached and a black hole is formed.

In the next sections, we will first discuss the behavior of the condensate with baryonic matter in the diluted approximation, when we can neglect the local reaction of the condensate. When we go beyond this approximation, we have to take into account the reaction of the cosmic condensate to the presence of the baryonic matter. We will see that this can be described as a dark force, mediated by gravitons pulled out from the cosmic BEC at galactic scales, which can explain the phenomenology at galactic scales commonly attributed to dark matter.

An important point to be stressed is that the dark force is a local effect. The cosmological BEC at horizon scales $L$ remains largely unaffected. This means that deviations from Eq. (2.11) for the cosmological BEC remain negligible at present, albeit they are crucial in order to describe the local dynamics properly, as we are going to start showing next. Our description is consistent as long as we are only concerned with the gravitational dynamics at galactic scales and we do not use our model to describe the whole cosmological history of our Universe. In order to do this, it is likely that more input is needed.

\section{BARYONIC MATTER IN THE DILUTED APPROXIMATION}

We now want to see in more detail what happens when very diluted baryonic matter is formed on top of the condensate of gravitons. In this approximation, matter can be considered as being made of, say, $N_{\mu}$ almost pointlike sources of mass $\mu$, at rest and equally distanced very far apart. We can therefore neglect the local reaction of the condensate to their presence, which also means that the gravitational interactions among matter sources are negligible. Since sources are homogeneously distributed, our results should also be a good approximation for baryonic matter with homogeneous density. We will see that the leading-order effect of baryonic matter is to subtract gravitons from the condensate.

\section{A. Diluted matter in the de Sitter universe}

Let us first see what happens when we introduce baryonic matter into the de Sitter universe, whose metric takes the form

$$
\mathrm{d} s^{2}=-f(r) \mathrm{d} t^{2}+f^{-1}(r) \mathrm{d} r^{2}+r^{2} \mathrm{~d} \Omega^{2} .
$$

Since in the diluted approximation the cumulative effect of many sources is just the sum of the single contributions, we start by considering the case of a single pointlike source of mass $\mu$. In the weak field regime, the metric function in Eq. (3.1) is given by the Schwarzschild-dS form

$$
f(r)=1-\frac{r^{2}}{L^{2}}+2 \phi(r),
$$

where

$$
\phi(r)=-\frac{G_{\mathrm{N}} \mu}{r}
$$

is the Newtonian potential generated by the source of mass $\mu$. The size $L_{H}$ of the cosmological horizon can be found by solving the condition $f(r)=0$ for small departures from $L$ (i.e. for $|\phi| \ll 1$ ), which yields

$$
L_{H}=L[1+\phi(L)]+o\left(\phi^{2}\right) \sim L-\ell_{\mathrm{p}} \mu / m_{\mathrm{p}}
$$

Adding $N_{\mu}$ similar matter sources would reduce the Hubble radius to

$$
L_{H} \sim L-\frac{1}{2} N_{\mu} R_{\mathrm{H}},
$$

where here $R_{\mathrm{H}}=2 G_{\mathrm{N}} \mu$ is the typical gravitational radius of each source. The effect of the presence of diluted matter is thus to reduce the size of the cosmological horizon, which in turn implies a number of gravitons in the cosmological condensate $N_{\mathrm{G}}<N_{\Lambda}$ according to Eq. (2.11). Let us note, 
however, that such a change is relatively minuscule because of the hierarchy (2.3), and the fact that baryonic matter accounts for at most $5 \%$ of the total energy in the universe. We can therefore safely neglect the difference between $L_{H}$ and $L$ in the following.

\section{B. Diluted matter in the corpuscular model}

Before we introduce the diluted baryonic matter in the DEC, let us refine the corpuscular description of the dS universe. In Refs. [39,40], it was shown that the maximal packing condition which yields the scaling relations (2.14) for a black hole actually follows from the energy balance (2.1) when matter becomes totally negligible. In the present case, matter is absent a priori and $H_{\mathrm{B}}=0$, so that one is left with

$$
H_{\mathrm{G}}^{(0)}=U_{\mathrm{N}}^{(0)}+U_{\mathrm{PN}}^{(0)}=0,
$$

with the negative Newtonian energy

$$
U_{\mathrm{N}}^{(0)} \simeq N_{\Lambda} \varepsilon_{\Lambda}=-N_{\Lambda} \frac{\ell_{\mathrm{p}} m_{\mathrm{p}}}{L},
$$

and the positive "post-Newtonian" contribution

$$
U_{\mathrm{PN}}^{(0)}=N_{\Lambda} \frac{\sqrt{N_{\Lambda}} \ell_{\mathrm{p}}^{2} m_{\mathrm{p}}}{L^{2}} .
$$

One therefore recovers the scaling relation (2.11) from Eq. (3.6) with no extra "vacuum energy" [40].

The same result (3.5) can now be obtained using the Hamiltonian constraint (2.1) in which we include the contribution of $N_{\mu}$ diluted baryonic sources of mass $\mu$,

$$
H_{\mathrm{B}}^{(1)}=N_{\mu} \mu
$$

Since matter is very diluted and cold, $\mu$ again just equals the proper mass, and local gravitational energy is negligible. We can therefore write

$$
H_{\mathrm{G}}^{(1)}=U_{\mathrm{N}}^{(1)}+U_{\mathrm{PN}}^{(1)},
$$

where the Newtonian and post-Newtonian terms have the forms given in Eqs. (3.7) and (3.8). The energy balance (2.1) then tells us that the condensate must respond to the presence of this homogeneous matter by changing the graviton number $N_{\Lambda}$, that is

$$
N_{\Lambda} \frac{\ell_{\mathrm{p}} m_{\mathrm{p}}}{L} \simeq N_{\mu} \mu+N_{\Lambda}^{3 / 2} \frac{\ell_{\mathrm{p}}^{2} m_{\mathrm{p}}}{L^{2}},
$$

which yields

$$
\begin{aligned}
L & =\frac{\ell_{\mathrm{p}} m_{\mathrm{p}} N_{\Lambda}}{2 N_{\mu} \mu}\left(1-\sqrt{1-\frac{4 N_{\mu} \mu}{\sqrt{N_{\Lambda}} m_{\mathrm{p}}}}\right) \\
& \simeq \sqrt{N_{\Lambda}} \ell_{\mathrm{p}}+N_{\mu} \ell_{\mathrm{p}} \mu / m_{\mathrm{p}}+\mathcal{O}\left(N_{\Lambda}^{-1}\right),
\end{aligned}
$$

where we used $N_{\mu} \mu \ll N_{\Lambda} m_{\mathrm{p}}$ for our dark energy dominated universe. Using now the scaling (2.11), i.e. $N_{\Lambda} \sim L_{H}^{2} / \ell_{\mathrm{p}}^{2}$, one easily recovers Eq. (3.5).

The fact that the two estimates, respectively based on the form of the Schwarzschild-dS metric and on the corpuscular model, give the same result for the change of the Hubble horizon due to the presence of baryonic matter is a highly nontrivial check of the validity of our BEC description of the dS universe, and in particular of the validity of the energy balance (3.10) and of the form of the postNewtonian term $U_{\mathrm{PN}}$.

\section{Diluted matter and scalings of the graviton number}

The change in the dS horizon size (3.5) induced by the baryonic matter will result in a reduction of the number of gravitons $N_{\Lambda}$ with energy $\varepsilon_{\Lambda}$ according to Eq. (2.11), that is

$$
\delta N_{\Lambda} \simeq-\frac{2 \mu L}{m_{\mathrm{p}} \ell_{\mathrm{p}}},
$$

where $N_{\Lambda}$ is given in (2.11). The same result holds also for a black hole of mass $\mu$, with $L$ replaced with the black hole radius $R_{\mathrm{H}}$ [3]. Actually, this result is a quite generic consequence of the holographic scaling (2.14) for the graviton number. In fact, let us take a sphere of radius $r \ll L$, for which the number of gravitons in the condensate inside this sphere is given by Eq. (2.14), and compare the change of the graviton number as a function of the radial distance from the center of the sphere with and without matter. Without the mass, the radial distance $s$ is equal to $r$, whereas a baryonic pointlike mass $\mu$ at the center of the sphere changes the radial distance of a quantity equal to $\mathrm{d} s \simeq[1-\phi(r)] \mathrm{d} r$, according to the weak field limit of the Schwarzschild metric. Thus, the number of gravitons in Eq. (2.14) changes due to the presence of matter according to

$\frac{\mathrm{d}\left(\delta N_{\Lambda}\right)}{\mathrm{d} s}=\frac{\mathrm{d}}{\mathrm{d} s}\left(\left.N_{\Lambda}\right|_{\mu \neq 0}-\left.N_{\Lambda}\right|_{\mu=0}\right) \simeq \phi(r) \frac{\mathrm{d} N_{\Lambda}}{\mathrm{d} r} \simeq-\frac{2 \mu}{m_{\mathrm{p}} \ell_{\mathrm{p}}}$.

On the other hand, in the diluted approximation $|\phi(r)| \ll 1$ and $\mathrm{d} r \simeq \mathrm{d} s$. We can thus write the previous equation as

$$
\frac{\mathrm{d}\left(\delta N_{\Lambda}\right)}{\mathrm{d} r} \simeq-\frac{2 \mu}{m_{\mathrm{p}} \ell_{\mathrm{p}}}
$$

For future convenience, we will define $N_{\mathrm{B}}=-\delta N_{\Lambda}$ as the number of gravitons subtracted from the cosmological condensate (the DEC) inside a sphere of radius $r$ by the 
presence of the baryonic source of mass $\mu$. By integrating the above equation, one finds

$$
N_{\mathrm{B}} \simeq-\frac{2 \mu r}{m_{\mathrm{p}} \ell_{\mathrm{p}}} .
$$

Extending the validity of Eq. (3.5) from the cosmological horizon to a region of radius $r$ as given in Eq. (3.14) is a quite strong and highly nontrivial assumption. In the corpuscular description of gravity, this implies that we are assuming not only that the whole dS space filled with dark energy can be considered as a graviton condensate with Compton length $L$, but that this description also holds for regions of any size $r$, and for those gravitons with Compton length of order $r$. The rationale behind this assumption is the fact that at solar system scales we know that gravity is well described by GR, whose action is directly related with the holographic scaling (2.14). It should be stressed that this holds only in the holographic regime of gravity (2.14) but not in the extensive regime (2.18). This means that our Universe looks like a critical graviton condensate at small (solar system) scales and very large (Hubble radius) scales, whereas at intermediate (galactic) scales we see an extensive behavior. In order to give a precise meaning for the transition from cosmological to intermediate scales, we can use arguments similar to those used by Verlinde in Ref. [3].

We suppose that, as shown in [3], the "dark matter" effects arise from the competition between the "area-law" (2.14) and volume behavior (2.18) for the graviton number. This implies the existence of two regimes: the baryonic matter dominated regime in which $N_{\mathrm{B}}(r)>N_{\mathrm{G}}^{V}(r)$ and a dark energy dominated regime $N_{\mathrm{B}}(r)<N_{\mathrm{G}}^{V}(r)$. In particular, we expect the dark force effects to be negligible for $N_{\mathrm{B}}(r) \gg N_{\mathrm{G}}^{V}(r)$. Let us now look for the transition between these two regimes, when the corresponding graviton numbers become comparable, that is $\left|N_{\mathrm{B}}(r)\right| \simeq$ $N_{\mathrm{G}}^{V}(r)$, or

$$
\frac{2 \mu r}{m_{\mathrm{p}} \ell_{\mathrm{p}}} \simeq \frac{r^{3}}{\ell_{\mathrm{p}}^{2} L}
$$

When this equality holds, most of the dark energy gravitons in the cosmological condensate contained inside the volume of size $r$ are affected by the presence of the source of mass $\mu$, and we obtain

$$
r \equiv r_{0} \simeq \sqrt{\frac{2 \mu}{m_{\mathrm{p}}} L \ell_{\mathrm{p}}}=\sqrt{R_{\mathrm{H}} L},
$$

where $r_{0}$ is the mesoscopic scale introduced in Sec. II B. For a given (spherical) region with a certain amount of mass $\mu$ localized about its center, $r_{0}$ sets the scale at which dark matter phenomena are not negligible. Using for $\mu$ the value for the mass of a galaxy in Eq. (3.17), one finds the observationally correct order of magnitude for deviations from the Newtonian dynamics. For instance, for a typical spiral galaxy with $m_{\mathrm{B}}=10^{11}$ solar masses, we have $r_{0}=6 \mathrm{kpc}$, whereas for a typical dwarf galaxy with $m_{\mathrm{B}}=$ $10^{7}$ solar masses, we have $r_{0}=80 \mathrm{pc}$.

To describe the transition between the holographic and the extensive regimes, it is convenient to introduce the local (size-dependent) parameter

$$
\gamma=\frac{N_{\mathrm{B}}}{N_{\mathrm{G}}^{V}} .
$$

For $\gamma>1$, we are in the area-scaling regime (2.14), where baryonic matter dominates, gravity is well described by $\mathrm{GR}$, and most of the gravitons in the fluid belong to the condensate. Conversely, for $\gamma<1$, we are in the volumescaling regime (2.14) and dark energy dominates. In this regime, the effects of the dark energy gravitons on baryonic matter are not negligible and give rise to the dark matter phenomena.

Let us conclude with some comments about the physical meaning of the diluted approximation and on the meaning of Eq. (3.19). Within this approximation, baryonic matter has no local gravitational interactions with the condensate. On the other hand, it also has no effects at cosmological scales. Rephrased in terms of the graviton number, the diluted regime applies in the region where $\gamma>1$, i.e. when most gravitons inside a sphere of radius $r$ belong to the local condensate (we are considering the sphere of radius $r$ as a condensate of gravitons of Compton length $r$ ).

\section{CLUMPED MATTER AND EMERGENCE OF THE DARK FORCE}

Let us now describe what happens when we go beyond the diluted approximation and baryonic matter begins to clump. The $N_{\mu}$ pointlike sources of mass $\mu$ form clusters of baryonic matter with typical mass $m_{\mathrm{B}}(r)=N_{\mu} \mu$. For simplicity, we consider a mass distribution with spherical symmetry. Now the DEC will react to the presence of matter, and we will interpret this reaction as a dark force responsible for the phenomenology commonly attributed to dark matter which reproduces correctly the MOND acceleration.

We first assume that only a fraction of the gravitons in the DEC are affected by the local matter, so that the condensate reacts not at the full cosmological scale $L$, but at a local scale of size $r$. In particular, since we are considering spherically symmetric sources, the baryonic matter of mass $m_{\mathrm{B}}$ will pull the gravitons out of the DEC from inside the sphere of radius $r$ with a dark energy mass given by $M=M(r)$. Therefore we now have three scales in our problem: the typical size of the matter lumps $R_{\mathrm{B}}$, the range of the condensate reaction $r$ and $L$, which satisfy the hierarchy 


$$
R_{\mathrm{H}} \ll R_{\mathrm{B}} \lesssim r \ll L
$$

In the following we will consider the dynamics of test particles at distances $r \gg R_{\mathrm{B}}$, so that the baryonic source of mass $m_{\mathrm{B}}(r)$ can be well approximated by a pointlike source. Physically, this means that we are considering the dynamics of galaxies at distances far away from the galactic core. We will first briefly review the results of Ref. [38] based on a balance between the number of gravitons, we will then give the description based on the Hamiltonian constraint, and finally we will proceed by using the competition between the area and volume regimes to derive the "dark acceleration."

\section{A. Matter clumping and graviton number balance}

The starting point of the analysis in Ref. [38] is that, in a corpuscular description of gravity, the gravitational acceleration felt by the test particle is the macroscopic manifestation of the self-interaction of gravitons in the condensate. It can therefore be expressed in terms of their Compton energy $\varepsilon$ and specific number $N_{\text {eff }}$ of gravitons involved in the process as

$$
a(r) \simeq \frac{\varepsilon^{2}(r)}{m_{\mathrm{p}}^{2} \ell_{\mathrm{p}}} \sqrt{N_{\mathrm{eff}}} .
$$

Moreover, this corpuscular acceleration formula holds for both condensed and noncondensed gravitons. Consider now the reaction of the cosmological BEC of total mass $m_{\Lambda}$ to the presence of the baryonic matter source of mass $m_{\mathrm{B}}(r)$. Since $m_{\mathrm{B}} \ll m_{\Lambda}$, most of the gravitons will remain in the condensed phase and their number is given, according to Eq. (2.14), by

$$
N_{\mathrm{DE}} \sim \frac{\left(m_{\Lambda}-m_{B}\right)^{2}}{m_{\mathrm{p}}^{2}} .
$$

On the other hand, the total number of gravitons in the system is given by $N_{\Lambda} \sim m_{\Lambda}^{2} / m_{\mathrm{p}}^{2}$. This implies that there are $N_{\Lambda}-N_{\text {DE }}$ gravitons which are not in the condensed phase and, therefore, behave differently from the condensate. Since the number of gravitons which give rise to the local gravitational potential generated by the baryonic mass is $N_{\mathrm{B}}=m_{B}^{2} / m_{\mathrm{p}}^{2}$ and, from Eqs. (4.3), we have

$$
N_{\Lambda}-N_{\mathrm{DE}} \sim \frac{L m_{\mathrm{B}}}{\ell_{\mathrm{p}} m_{\mathrm{p}}}-\frac{m_{B}^{2}}{m_{\mathrm{p}}^{2}}
$$

it follows that there are $N_{\mathrm{DF}} \sim L m_{\mathrm{B}} / \ell_{\mathrm{p}} m_{\mathrm{p}}$ gravitons which mediate the interaction between the baryonic matter and the DEC.

The effective number of noncondensed gravitons $N_{\mathrm{DF}}(r)$ that contribute to the acceleration of a test particle at the radius $r$ can be guessed by requiring that its overall scaling is again holographic and must depend on the baryonic mass $m_{\mathrm{B}}$. This yields

$$
N_{\mathrm{DF}}(r) \sim \frac{r^{2} m_{\mathrm{B}}(r)}{\ell_{\mathrm{p}} m_{\mathrm{p}} L}
$$

From Eqs. (4.2) and (4.5) with $N_{\text {eff }}=N_{\mathrm{DF}}(r)$, we obtain

$$
\left|a_{\mathrm{DF}}(r)\right| \sim \sqrt{\frac{G_{\mathrm{N}} m_{\mathrm{B}}(r)}{L r^{2}}} \sim \sqrt{\frac{a_{\mathrm{B}}(r)}{L}}
$$

which is the MOND acceleration (1.2) up to a numerical factor.

\section{B. Matter clumping and energy balance}

In this section we will alternatively derive the MOND acceleration (1.2) using the Hamiltonian constraint (2.1).

Once the regular matter starts clumping, the matter energy changes to

$$
H_{\mathrm{B}}=m_{\mathrm{B}}+E_{\mathrm{B}}
$$

where $m_{\mathrm{B}} \simeq N_{\mu} \mu$ and $E_{\mathrm{B}}$ accounts for the total kinetic energy of matter and nongravitational interactions. Some gravitons will acquire a new Compton length in response to the local lumps of matter, and the gravitational Hamiltonian in the constraint Eq. (2.1) takes the form

$$
H_{\mathrm{G}}=H_{\Lambda}+H_{\mathrm{BG}}+H_{\mathrm{DF}}
$$

where $H_{\Lambda}$ is the energy of the DEC, whose specific form is not essential for the present derivation; $H_{\mathrm{BG}}$ is the Newtonian gravitational energy of the localized matter sources,

$$
H_{\mathrm{BG}}=-\frac{G_{\mathrm{N}} m_{\mathrm{B}}^{2}}{R_{\mathrm{B}}}=-N_{\mathrm{B}} \frac{\ell_{\mathrm{p}} m_{\mathrm{p}}}{R_{\mathrm{B}}},
$$

with $N_{\mathrm{B}}$ the number of soft gravitons whose Compton length equals the typical size $R_{\mathrm{B}}$ of matter lumps ${ }^{4}$ finally, the "dark force" term is given by the gravitational interaction energy between baryonic matter and dark energy of mass $M(r)$ inside the sphere of radius $r$, that is

$$
H_{\mathrm{DF}}=-\frac{G_{\mathrm{N}} m_{\mathrm{B}} M(r)}{r} .
$$

We can rewrite $H_{\mathrm{DF}}$ in terms of an effective dark force mass $m_{\mathrm{DF}}$ as

\footnotetext{
${ }^{4}$ There would also be a (positive) post-Newtonian energy but we shall neglect that as it is much smaller than $H_{\mathrm{BG}}$ for compact sources far from becoming black holes.
} 


$$
\frac{G_{\mathrm{N}} m_{\mathrm{B}} M(r)}{r} \simeq \frac{G_{\mathrm{N}} m_{\mathrm{DF}}^{2}}{r},
$$

which implies the simple relation between masses

$$
m_{\mathrm{DF}}^{2}=m_{\mathrm{B}} M(r) .
$$

Because the dark matter term arises from the interaction of the baryonic source with the gravitons in the DEC inside the volume of size $r$, the energy of the gravitons will change to $\varepsilon \simeq m_{\mathrm{p}} \ell_{\mathrm{p}} / r$. From the extensive scaling (2.18), it follows that

$$
M(r) \simeq \frac{m_{\mathrm{p}} r^{2}}{\ell_{\mathrm{p}} L}
$$

We can now evaluate the gravitational acceleration associated to the dark force component (4.11) of the condensate. Using the estimate (4.13) in Eq. (4.12), we obtain the dark acceleration

$$
a_{\mathrm{DF}} \sim \frac{G_{\mathrm{N}} m_{\mathrm{DM}}}{r^{2}} \simeq \sqrt{\frac{1}{L} \frac{G_{\mathrm{N}} m_{\mathrm{B}}}{r^{2}}}=\sqrt{\frac{a_{\mathrm{B}}(r)}{L}},
$$

where $a_{\mathrm{B}}(r)$ is the Newtonian baryonic acceleration (1.3) at distances $r$. Again, this result indeed matches the MOND formula (1.2) up to a factor of $1 / 6$.

We can further show that the above derivation, based on the energy balance (4.8), is perfectly compatible and consistent with the derivation in Sec. IV A, which is instead based on the graviton numbers. In fact, we can associate to the dark energy mass $M(r)$ interacting with the baryonic mass a number of gravitons equal to the number of gravitons $N_{\mathrm{DF}}(r)$ pulled out from the DEC. This number scales holographically as

$$
N_{\mathrm{DF}}(r)=\frac{m_{\mathrm{DF}}^{2}}{m_{p}^{2}} .
$$

By combining Eqs. (4.12) and (4.15), we find

$$
M(r)=N_{\mathrm{DF}}(r) \frac{m_{\mathrm{p}}^{2}}{m_{\mathrm{B}}},
$$

and Eq. (4.13) finally yields the total number of gravitons associated to the dark force

$$
N_{\mathrm{DF}}(r)=\frac{m_{\mathrm{B}} r^{2}}{\ell_{\mathrm{p}} m_{\mathrm{p}} L},
$$

which exactly matches Eq. (4.5) obtained in Sec. IVA.

We further note that the dark acceleration can also be written as a function of the number of dark gravitons, thus obtaining the same expression (4.2) found in Sec. IVA. In fact, by combining Eqs. (4.16) and (4.12), we find

$$
a_{\mathrm{DF}}=\frac{G_{\mathrm{N}} m_{\mathrm{DF}}}{r^{2}}=\frac{\ell_{\mathrm{p}}}{r^{2}} \sqrt{N_{\mathrm{DF}}(r)},
$$

or, equivalently, using the Compton energy of the dark gravitons $\varepsilon=m_{\mathrm{p}} \ell_{\mathrm{p}} / r$,

$$
a_{\mathrm{DF}}=\frac{\varepsilon^{2}(r)}{m_{\mathrm{p}}^{2} \ell_{\mathrm{p}}} \sqrt{N_{\mathrm{DF}}(r)},
$$

which is exactly the corpuscular acceleration (4.2) first introduced in Ref. [38] for $N_{\text {eff }}=N_{\mathrm{DF}}(r)$.

\section{Area/volume competition and heuristic derivation of MOND}

In this section, we present a heuristic derivation of the MOND acceleration (1.2), which uses the Hamiltonian constraint (4.8) and the competition between the holographic and extensive regimes described in Sec. II. The novelty is that we will be able to reproduce correctly also the numerical factors of Eq. (1.2) in this scenario. The key observation is that, owing to the fact that the DEC responds only locally to the presence of baryonic matter, we can simply write the contribution $H_{\mathrm{DF}}$ in Eq. (4.8) in terms of the energy subtracted from dark energy gravitons to generate the local Newtonian gravity.

For simplicity, we consider baryonic matter in the form of a single pointlike source of mass $m_{\mathrm{B}}$, but the results can be easily generalized to the case of an extended but localized source inside a volume of size $R_{\mathrm{B}}$. By analogy with the electromagnetic force, the energy density $\rho_{\mathrm{G}}$ associated with a gravitational (acceleration) field (1.3), that is

$$
a_{\mathrm{B}}=-\frac{G_{\mathrm{N}} m_{\mathrm{B}}}{r^{2}},
$$

inside a sphere of radius $r$, and volume $V(r)=4 \pi r^{3} / 3$, is given by

$$
\rho_{\mathrm{G}}=\frac{a_{B}^{2}}{8 \pi G_{\mathrm{N}}},
$$

where $m_{\mathrm{B}}$ is the source of the gravitational field. It is easy to find that the energy subtracted from dark energy gravitons in order to clump the amount of matter $m_{\mathrm{B}}$ inside the spherical region is, therefore,

$$
E_{\mathrm{G}}=-\rho_{\mathrm{G}} V=-\frac{G_{\mathrm{N}} m_{\mathrm{B}}^{2}}{6 r},
$$

where $m_{\mathrm{B}}$ now denotes the baryonic mass contained inside $V(r)$.

Consistently with Eq. (4.12), we can view this energy as due to the existence of a dark force, whose effective source is a "dark mass" $m_{\mathrm{DF}}$, which does work on the system. In analogy to what happens at cosmological scales, we can think that the effect of the mass $m_{\mathrm{B}}$, centered inside a 
spherical region of volume $V(r)$, is to deform the sphere by an amount given by Eq. (3.5) with $L$ replaced by $r$. The deformation is therefore

$$
u(r)=\phi_{\mathrm{B}}(r) L,
$$

where $\phi_{\mathrm{B}}$ is the gravitational potential generated by the mass $m_{\mathrm{B}}$ and $L$ is still the $\mathrm{dS}$ radius. The work done by the dark force on the system will be given by

$$
W=F_{\mathrm{DF}} u(r)=\frac{G_{\mathrm{N}}^{2} m_{\mathrm{DF}}^{2} m_{\mathrm{B}}}{r^{3}} L .
$$

It should be stressed that this contribution is of a holographic nature: it is the work done by the dark force to deform the surface of the sphere. For energy conservation, it must equal the energy $E_{\mathrm{G}}$ contained in the volume $V(r)$. By equating Eqs. (4.22) and (4.24), we easily obtain

$$
\frac{G_{\mathrm{N}} m_{\mathrm{DF}}^{2}}{r^{2}}=-\frac{m_{\mathrm{B}}}{6 L} .
$$

If we now use the form for the "dark gravitational acceleration" used in (4.14),

$$
a_{\mathrm{DF}}=-\frac{G_{\mathrm{N}} m_{\mathrm{DF}}}{r^{2}}
$$

and the Newtonian acceleration (4.20), Eq. (4.25) can be written as

$$
a_{\mathrm{DF}}(r)=\sqrt{\frac{a_{\mathrm{B}}}{6 L}}
$$

which exactly matches the MOND acceleration (1.2). Let us stress that Eq. (1.2) is precisely obtained by identifying the volume (extensive) subtraction (4.22) from the condensate with the dark area (holographic) contribution (4.24).

\section{Emergent metric theory}

A key issue for every model of emergent gravity is the existence of an effective description reproducing Einstein's GR or at least a metric theory of gravity. One must envisage the way in which the metric space-time structure of gravity encoded in GR emerges out of the microscopic description. This is a quite stringent requirement and it is not enough to predict an infrared modification of the laws of gravity, such as the MOND relation (1.2). This relation must be embedded in the framework of GR or, at least, in a metric theory of gravity describing a modification thereof. This is for instance a drawback of Verlinde's original proposal [3]. The proposed modification of the laws of gravity at galactic scales reproduces the MOND relation (1.2), but a metric covariant description of the model has not been proposed yet (see, however, Refs. [48-50]).

The description of the emergent laws of gravity at galactic scales based on the BEC of gravitons proposed in this paper allows for an effective covariant metric description, which has the form of GR sourced by an anisotropic fluid. This can be done along the lines of Refs. [38,51]. We know that a dark energy dominated universe, i.e. the DEC of gravitons, can be described in a metric framework as GR sourced by a perfect fluid with constant energy density $\rho$ and equation of state $p=-\rho$. The generation of baryonic matter and the reaction of the condensate allows for an effective description in which the fluid becomes anisotropic. In this effective fluid description, the dark acceleration $a_{\mathrm{DF}}$ is completely due to the pressure of the anisotropic fluid $p_{\|_{\mathrm{DF}}}(r)$. The (modulus of the) total acceleration experienced by a test particle is given by [38]

$$
a_{\mathrm{B}}+a_{\mathrm{DF}} \simeq \frac{G_{\mathrm{N}} m_{\mathrm{B}}(r)}{r^{2}}+4 \pi G_{\mathrm{N}} r p_{\|_{\mathrm{DF}}}(r) .
$$

Solving Einstein equations sourced by the anisotropic fluid, one finds the space-time metric in the form

$$
\mathrm{d} s^{2}=-f(r) e^{\gamma(r)} \mathrm{d} t^{2}+\frac{\mathrm{d} r^{2}}{f(r)}+r^{2} \mathrm{~d} \Omega^{2},
$$

with

$$
f(r)=1-\frac{2 G_{\mathrm{N}} m(r)}{r},
$$

and the metric function $\gamma$ determined by the distribution of baryonic matter $m_{\mathrm{B}}$,

$$
\gamma^{\prime}=\frac{2}{r f(r)}\left[G_{\mathrm{N}} m_{\mathrm{B}}^{\prime}(r)+\sqrt{a_{0} G_{\mathrm{N}} m_{\mathrm{B}}(r)}\right] .
$$

It has also been shown that, when we can approximate the baryonic mass distribution with a constant profile $m_{\mathrm{B}}(r)=$ $m_{\mathrm{B}}$ (this holds when we consider a galaxy at distances much bigger than its bulk), we recover the typical logarithmic behavior of the MOND gravitational potential [38] and Tully-Fisher relation.

\section{E. Cosmic balance}

If one puts together the argument based on the graviton number of Sec. IV A and the energy balance of Sec. IV B, the ratio between an apparent dark matter mass distribution and baryonic matter can be estimated and shown to be consistent with the predictions of the $\Lambda \mathrm{CDM}$ model.

Let us denote with $U_{\mathrm{DF}}$ the energy associated with the dark gravitons. This energy can be written in terms of the number $N_{\mathrm{DF}}$ of dark force gravitons inside a sphere of radius $r$ and their Compton energy $\varepsilon=-m_{\mathrm{p}} \ell_{\mathrm{p}} / r$ as

$$
U_{\mathrm{DF}}=N_{\mathrm{DF}} \varepsilon=-N_{\mathrm{DF}} \frac{m_{\mathrm{p}} \ell_{\mathrm{p}}}{r} .
$$

In the $\Lambda \mathrm{CDM}$ description, $U_{\mathrm{DF}}$ must be seen as originating from the interaction of an apparent dark matter mass $M_{\mathrm{DM}}$ 
with the baryonic matter of mass $m_{\mathrm{B}}$ and its selfinteraction, that is

$$
U_{\mathrm{DF}}=-\frac{G_{\mathrm{N}} m_{\mathrm{DM}} m_{\mathrm{B}}}{r}-\frac{G_{\mathrm{N}} m_{\mathrm{DM}}^{2}}{r} .
$$

Equating the above two expressions for $U_{\mathrm{DF}}$, we get

$$
N_{\mathrm{DF}}=\frac{m_{\mathrm{DM}}^{2}}{m_{\mathrm{p}}^{2}}+\frac{m_{\mathrm{DM}} m_{\mathrm{B}}}{m_{\mathrm{p}}^{2}} .
$$

Let us stress that the apparent dark matter mass $m_{\mathrm{DM}}$ must not be confused with the effective dark force mass $m_{\mathrm{DF}}$ of Eq. (4.12). In fact, consistency of Eq. (4.34) with Eq. (4.15) requires $m_{\mathrm{DF}}^{2}=m_{\mathrm{DM}}^{2}+m_{\mathrm{DM}} m_{B}$.

On using Eq. (4.3), $N_{\mathrm{DF}} \sim N_{\Lambda}-N_{\mathrm{DE}}$, and recalling that $N_{\Lambda} \sim L^{2} / \ell_{\mathrm{p}}^{2}$ and $m_{\Lambda}=m_{\mathrm{p}} L / \ell_{\mathrm{p}}$, we obtain

$$
m_{\mathrm{DM}} m_{\mathrm{B}}+m_{\mathrm{DM}}^{2}=2 m_{\Lambda} m_{\mathrm{B}}-m_{\mathrm{B}}^{2},
$$

which can be written as

$$
x^{2}+x+1=\frac{2 m_{\Lambda}}{m_{\mathrm{B}}},
$$

where we defined the ratio $x=m_{\mathrm{DM}} / m_{\mathrm{B}}$. In particular, the latter equation is solved by

$$
\frac{m_{\mathrm{DM}}}{m_{\mathrm{B}}}=\frac{\sqrt{8\left(m_{\Lambda} / m_{\mathrm{B}}\right)-3}-1}{2} .
$$

If we now recall that observations yield $m_{\mathrm{B}} \simeq 0.05 m_{\Lambda}$, we finally obtain

$$
\frac{m_{\mathrm{DM}}}{m_{\mathrm{B}}} \simeq 5.77,
$$

which is in the right ballpark of the $\Lambda \mathrm{CDM}$ prediction for the present relative abundance of dark and baryonic matter.

\section{CONCLUSIONS}

In this work, we have investigated the emergent laws of gravity by modeling our dark energy dominated universe as a critical BEC with a large number $N_{\mathrm{G}}$ of soft gravitons. We have shown that the local behavior of this DEC requires, besides the usual holographic regime, an extensive regime of gravity in which the graviton number scales with the volume of space. Baryonic matter fits naturally in this description as gravitons pulled out from the DEC at the matter clumping scale give rise to the local (Newtonian) gravitational forces. We have then shown that, in this framework, the galaxy rotation curves far away from the galactic center [i.e. the MOND formula (1.2)] can likewise be derived from the reaction of the DEC to the presence of baryonic matter, without assuming the existence of any sort of dark matter. We have also evaluated the mass ratio of the apparent dark matter and baryonic component and found it to be in agreement with the prediction of the $\Lambda \mathrm{CDM}$ model.
Moreover, our microscopic description can be easily used to produce an emergent theory of gravity in the form of GR sourced by an anisotropic fluid, the latter being the macroscopic manifestation of the DEC and of its interaction with baryonic matter. This can be done along the lines of Refs. [38,51], in which the dark force explaining galactic dynamics furthermore takes the form of a pure pressure term [38].

We would like to conclude by remarking that two important points have not yet been addressed, but deserve further investigation. The first one concerns the microscopic origin of the cosmological evolution. Our model applies solely to the present dark energy dominated universe. We did not tackle the problem of giving a description of the history of the universe using a critical BEC of soft gravitons. Although this is a quite involved problem, there are several indications that it may indeed be possible. The results of Refs. $[7,18,46]$ about the description of inflation and general cosmological space-times [41] represent promising steps along this direction. Moreover, the results of Ref. [47] not only assert that the $\mathrm{dS}$ space-time necessarily appears at late times in any cosmological evolution consistent with the generalized second law of thermodynamics, but also imply that the presence of an extensive, volume-scaling term for the graviton number is perfectly consistent with this late-time cosmological evolution. Last but not least, the fact that our model predicts the correct present relative abundance of the various forms of matter gives us a further hint that we are going in the right direction.

The second point concerns the microscopic origin of horizons. Most of the scenarios for emergent gravity assume in an explicit or implicit way the presence of event, cosmological or acceleration horizons (see, e.g., Ref. [52]). Horizons are a key ingredient for explaining the holographic regimes of gravity and play, therefore, a crucial role also in our BEC description of black holes in the dS space-time. At the level of the BEC, one may easily generate acoustic horizons [53]. However, it is not clear if acoustic horizons in a BEC can be directly linked to space-time horizons in the emergent gravity scenario. In fact, acoustic horizons in BEC are mainly of kinematic origin, whereas in an emergent gravity theory containing black holes and the dS space-time, their origin should be dynamical.

\section{ACKNOWLEDGMENTS}

We thank W. Mück for many useful discussions. A. G. is grateful to L. Berezhiani and A. Giugno for stimulating discussions. This research was partially supported by Istituto Nazionale di Fisica Nucleare (INFN), research initiatives FieLds And Gravity (FLAG) (R. C. and A. G.) and QUAntum GRAvity Phenomenology (QUAGRAP) (M. C. and M. T.). The work of R. C. and A. G. has been carried out in the framework of Gruppo Nazionale di Fisica Matematica (GNFM) and Istituto Nazionale di Alta Matematica (INdAM) and the european COoperation in Science and Thecnology (COST) action Cantata. 
[1] A. D. Sakharov, Vacuum quantum fluctuations in curved space and the theory of gravitation, Sov. Phys. Dokl. 12, 1040 (1968); Gen. Relativ. Gravit. 32, 365 (2000).

[2] T. Padmanabhan, Emergent gravity paradigm: Recent progress, Mod. Phys. Lett. A 30, 1540007 (2015).

[3] E. P. Verlinde, Emergent gravity and the dark universe, SciPost Phys. 2, 016 (2017).

[4] T. Padmanabhan, Thermodynamical aspects of gravity: New insights, Rep. Prog. Phys. 73, 046901 (2010).

[5] T. Padmanabhan, The atoms of space, gravity and the cosmological constant, Int. J. Mod. Phys. A D25, 1630020 (2016).

[6] G. Dvali and C. Gomez, Black hole's quantum N-portrait, Fortschr. Phys. 61, 742 (2013).

[7] G. Dvali and C. Gomez, Quantum compositeness of gravity: Black holes, AdS and inflation, J. Cosmol. Astropart. Phys. 01 (2014) 023.

[8] T. Jacobson, Thermodynamics of Space-Time: The Einstein Equation of State, Phys. Rev. Lett. 75, 1260 (1995).

[9] S. Bhattacharyya, V. E. Hubeny, S. Minwalla, and M. Rangamani, Nonlinear fluid dynamics from gravity, J. High Energy Phys. 02 (2008) 045.

[10] G. E. Volovik, Superfluid analogies of cosmological phenomena, Phys. Rep. 351, 195 (2001).

[11] J. D. Bekenstein, Black holes and entropy, Phys. Rev. D 7, 2333 (1973).

[12] J. M. Maldacena, The large N limit of superconformal field theories and supergravity, Int. J. Theor. Phys. 38, 1113 (1999); Adv. Theor. Math. Phys. 2, 231 (1998).

[13] S. Ryu and T. Takayanagi, Holographic Derivation of Entanglement Entropy from AdS/CFT, Phys. Rev. Lett. 96, 181602 (2006).

[14] M. Van Raamsdonk, Building up spacetime with quantum entanglement, Gen. Relativ. Gravit. 42, 2323 (2010); Int. J. Mod. Phys. A D19, 2429 (2010).

[15] T. Jacobson, Entanglement Equilibrium and the Einstein Equation, Phys. Rev. Lett. 116, 201101 (2016).

[16] G. Dvali and C. Gomez, Black hole's 1/N hair, Phys. Lett. B 719, 419 (2013).

[17] R. Casadio, A. Giugno, O. Micu, and A. Orlandi, Thermal BEC black holes, Entropy 17, 6893 (2015).

[18] R. Casadio, A. Giugno, and A. Giusti, Corpuscular slow-roll inflation, Phys. Rev. D 97, 024041 (2018).

[19] L. Berezhiani, On corpuscular theory of inflation, Eur. Phys. J. C 77, 106 (2017).

[20] A. Almheiri, D. Marolf, J. Polchinski, and J. Sully, Black holes: Complementarity or firewalls?, J. High Energy Phys. 02 (2013) 062.

[21] J. Maldacena and L. Susskind, Cool horizons for entangled black holes, Fortschr. Phys. 61, 781 (2013).

[22] F. Zwicky, Die Rotverschiebung von extragalaktischen Nebeln, Helv. Phys. Acta 6, 110 (1933); Gen. Relativ. Gravit. 41, 207 (2009).

[23] S. M. Faber and J. S. Gallagher, Masses and mass-to-light ratios of galaxies, Annu. Rev. Astron. Astrophys. 17, 135 (1979).

[24] J. de Swart, G. Bertone, and J. van Dongen, How dark matter came to matter, Nature Astron. 1, 0059 (2017).

[25] P. J. E. Peebles and B. Ratra, The cosmological constant and dark energy, Rev. Mod. Phys. 75, 559 (2003).
[26] A. G. Riess et al. (Supernova Search Team Collaboration), Observational evidence from supernovae for an accelerating universe and a cosmological constant, Astron. J. 116, 1009 (1998).

[27] A. A. Penzias and R. W. Wilson, A measurement of excess antenna temperature at 4080-Mc/s, Astrophys. J. 142, 415 (1965).

[28] P. A. R. Ade et al. (Planck Collaboration), Planck 2013 results. XVI. Cosmological parameters, Astron. Astrophys. 571, A16 (2014).

[29] A. A. Klypin, A. V. Kravtsov, O. Valenzuela, and F. Prada, Where are the missing Galactic satellites?, Astrophys. J. 522, 82 (1999).

[30] B. Moore, S. Ghigna, F. Governato, G. Lake, T. R. Quinn, J. Stadel, and P. Tozzi, Dark matter substructure within galactic halos, Astrophys. J. 524, L19 (1999).

[31] M. Boylan-Kolchin, J.S. Bullock, and M. Kaplinghat, Too big to fail? The puzzling darkness of massive Milky Way subhaloes, Mon. Not. R. Astron. Soc. 415, L40 (2011).

[32] M. Boylan-Kolchin, J. S. Bullock, and M. Kaplinghat, The Milky Way's bright satellites as an apparent failure of LCDM, Mon. Not. R. Astron. Soc. 422, 1203 (2012).

[33] V. C. Rubin, N. Thonnard, and W. K. Ford, Jr., Rotational properties of $21 \mathrm{SC}$ galaxies with a large range of luminosities and radii, from NGC $4605 / \mathrm{R}=4 \mathrm{kpc} /$ to UGC 2885/ $\mathrm{R}=122$ kpc/, Astrophys. J. 238, 471 (1980).

[34] M. Persic, P. Salucci, and F. Stel, The Universal rotation curve of spiral galaxies: 1. The dark matter connection, Mon. Not. R. Astron. Soc. 281, 27 (1996).

[35] R. B. Tully and J. R. Fisher, A new method of determining distances to galaxies, Astron. Astrophys. 54, 661 (1977).

[36] M. Milgrom, A modification of the Newtonian dynamics as a possible alternative to the hidden mass hypothesis, Astrophys. J. 270, 365 (1983).

[37] M. Milgrom, MOND theory, Can. J. Phys. 93, 107 (2015).

[38] M. Cadoni, R. Casadio, A. Giusti, W. Mueck, and M. Tuveri, Effective fluid description of the dark universe, Phys. Lett. B 776, 242 (2018).

[39] R. Casadio, A. Giugno, and A. Giusti, Matter and gravitons in the gravitational collapse, Phys. Lett. B 763, 337 (2016).

[40] R. Casadio, A. Giugno, A. Giusti, and M. Lenzi, Quantum corpuscular corrections to the Newtonian potential, Phys. Rev. D 96, 044010 (2017).

[41] P. Binetruy, Vacuum energy, holography and a quantum portrait of the visible Universe, arXiv:1208.4645.

[42] G. E. Volovik, Vacuum energy and cosmological constant: View from condensed matter, J. Low Temp. Phys. 124, 25 (2001).

[43] G. E. Volovik, The Universe in a helium droplet, Int. Ser. Monogr. Phys. 117, 1 (2006).

[44] G. E. Volovik, Cosmological constant and vacuum energy, Ann. Phys. (Amsterdam) 14, 165 (2005).

[45] M. Nishiyama, M.-a. Morita, and M. Morikawa, Bose Einstein condensation as dark energy and dark matter, arXiv:astro-ph/0403571.

[46] R. Casadio, F. Kuehnel, and A. Orlandi, Consistent cosmic microwave background spectra from quantum depletion, J. Cosmol. Astropart. Phys. 09 (2015) 002. 
[47] S. M. Carroll and A. Chatwin-Davies, Cosmic equilibration: A holographic no-hair theorem from the generalized second law, arXiv:1703.09241.

[48] S. Hossenfelder, Covariant version of Verlinde's emergent gravity, Phys. Rev. D 95, 124018 (2017).

[49] D.-C. Dai and D. Stojkovic, Comment on "Covariant version of Verlinde's emergent gravity”, Phys. Rev. D 96, 108501 (2017).

[50] R.-G. Cai, S. Sun, and Y.-L. Zhang, Emergent dark matter in late universe on holographic screen, arXiv:1712.09326.
[51] T. Faber and M. Visser, Combining rotation curves and gravitational lensing: How to measure the equation of state of dark matter in the galactic halo, Mon. Not. R. Astron. Soc. 372, 136 (2006).

[52] M. Cadoni and P. Jain, How is the presence of horizons and localized matter encoded in the entanglement entropy?, Int. J. Mod. Phys. A A32, 1750083 (2017).

[53] C. Barcelo, S. Liberati, and M. Visser, Analog gravity from Bose-Einstein condensates, Classical Quantum Gravity 18, 1137 (2001). 